\title{
Inhibition of transcription leads to rewiring of locus-specific chromatin proteomes
}

\author{
Deepani W. Poramba-Liyanage, ${ }^{1,6}$ Tessy Korthout, ${ }^{1,6}$ Christine E. Cucinotta, ${ }^{2}$ \\ Ila van Kruijsbergen, ${ }^{1}$ Tibor van Welsem, ${ }^{1}$ Dris El Atmioui, ${ }^{3,4}$ Huib Ovaa, ${ }^{3,4}$ \\ Toshio Tsukiyama, ${ }^{2}$ and Fred van Leeuwen ${ }^{1,5}$ \\ ${ }^{1}$ Division of Gene Regulation, Netherlands Cancer Institute, 1066CX Amsterdam, The Netherlands; ${ }^{2}$ Basic Sciences Division, Fred \\ Hutchinson Cancer Research Center, Seattle, Washington 98109, USA; ${ }^{3}$ Leiden Institute for Chemical Immunology, Leiden University \\ Medical Center, $2333 Z C$ Leiden, The Netherlands; ${ }^{4}$ Oncode Institute, Amsterdam University Medical Center, University of \\ Amsterdam, 1105 AZ Amsterdam, The Netherlands; ${ }^{5}$ Department of Medical Biology, Amsterdam University Medical Center, \\ University of Amsterdam, 1105 AZ Amsterdam, The Netherlands
}

\begin{abstract}
Transcription of a chromatin template involves the concerted interaction of many different proteins and protein complexes. Analyses of specific factors showed that these interactions change during stress and upon developmental switches. However, how the binding of multiple factors at any given locus is coordinated has been technically challenging to investigate. Here we used Epi-Decoder in yeast to systematically decode, at one transcribed locus, the chromatin binding changes of hundreds of proteins in parallel upon perturbation of transcription. By taking advantage of improved Epi-Decoder libraries, we observed broad rewiring of local chromatin proteomes following chemical inhibition of RNA polymerase. Rapid reduction of RNA polymerase II binding was accompanied by reduced binding of many other core transcription proteins and gain of chromatin remodelers. In quiescent cells, where strong transcriptional repression is induced by physiological signals, eviction of the core transcriptional machinery was accompanied by the appearance of quiescent cell-specific repressors and rewiring of the interactions of protein-folding factors and metabolic enzymes. These results show that Epi-Decoder provides a powerful strategy for capturing the temporal binding dynamics of multiple chromatin proteins under varying conditions and cell states. The systematic and comprehensive delineation of dynamic local chromatin proteomes will greatly aid in uncovering protein-protein relationships and protein functions at the chromatin template.
\end{abstract}

[Supplemental material is available for this article.]

Many proteins assemble on to DNA to implement gene regulatory programs and ensure the expression of a subset of genes in agreement with the state and environmental cues of the cell. RNA polymerase II (Pol II) is an integral part of this assembly as it catalyzes the DNA-dependent synthesis of messenger RNA (mRNA). Regulation of Pol II occurs at different stages of transcription and involves concerted actions of many proteins and protein complexes and dynamic post-translational modifications of histones and the carboxyl-terminal domain (CTD) of Pol II (Sainsbury et al. 2015; Chen et al. 2018; Li et al. 2018).

The process of transcription involves distinct stages: initiation, elongation, and termination (Sainsbury et al. 2015; Chen et al. 2018; Li et al. 2018). The wrapping of DNA by histones into nucleosomes presents a barrier for each of these steps. Chromatin remodelers recruited to promoters can displace nucleosomes to open up crucial recognition elements for transcription factor (TF) binding (Struhl and Segal 2013; Prasad et al. 2016). For initiation of transcription, Pol II assembles with basal TFs (TFIIB-H) at the promoter to form the preinitiation complex, which opens up the DNA, initiates RNA synthesis, and stimulates the escape of Pol II from the promoter (Sainsbury et al. 2015; Feng et al. 2016; Hantsche and Cramer 2017). Productive elongation in-

\footnotetext{
${ }^{6}$ These authors contributed equally to this work. Corresponding author: Fred.v.leeuwen@nki.nl Article published online before print. Article, supplemental material, and publication date are at http://www.genome.org/cgi/doi/10.1101/gr.256255.119. Freely available online through the Genome Research Open Access option.
}

volves recruitment of Pol II associated factor complex (Paf1C), DRB sensitivity inducing factor (DSIF), Spt4/5, Spt6, and facilitator of chromatin transcription (FACT) (Xu et al. 2017; Ehara and Sekine 2018; Vos et al. 2018). Toward the end of genes, transcription is terminated and the RNA is processed by the recruitment of cleavage and polyadenylation factors, which results in the release of Pol II and the nascent mRNA from the DNA template (Porrua and Libri 2015). Biochemical and genetic studies have provided a rich catalog of factors involved in the different stages of transcription. However, how the many different interactions are coordinated at any given chromatin locus in time and under changing conditions is still poorly understood (Qiu and Kaplan 2019).

Unraveling the relationships between proteins and the hierarchies among them at the chromatin template will require measuring the chromatin interactome and monitoring the changes upon perturbation. A common and convenient strategy to perturb transcription is the use of inhibitors of Pol II (Bensaude 2011). Some of the commonly used inhibitors of transcription initiation or elongation are not active in live yeast cells because of uptake deficiency or because the target protein or target site on the protein is not conserved (Bensaude 2011). However, the addition of the metal chelators thiolutin and 1,10-phenanthroline (PH) leads to rapid loss of transcription in yeast. This has, for example, enabled the determination of mRNA half-lives and establishing the role of Pol II activity in nucleosome positioning (Adams and Gross 1991;

(C) 2020 Poramba-Liyanage et al. This article, published in Genome Research, is available under a Creative Commons License (Attribution 4.0 International), as described at http://creativecommons.org/licenses/by/4.0/. 
Grigull et al. 2004; Sun et al. 2013; Hsieh et al. 2015; Vasseur et al. 2016; Lauinger et al. 2017). In addition, 6-azauracil (AU), which perturbs the supply of ribonucleotides and thereby indirectly affects RNA polymerases, is frequently used to assess transcription elongation phenotypes in yeast (Handschumacher and Welch 1956; Exinger and Lacroute 1992; Shaw and Reines 2000; Mason and Struhl 2005; Zhou et al. 2015). Although these chemicals and related compounds have been used extensively and are convenient and powerful tools to block transcription, little is known about how the direct and indirect inhibition of transcription affects the interactions of Pol II and its protein partners with the chromatin template.

Transcription is also regulated under physiological conditions during development and differentiation. In budding yeast, a global change in transcription is observed when cells enter quiescence, a survival mode that involves a G1-like cell-cycle arrest, with increased resistance to stress, metabolic rewiring, and largescale reorganization of the genome and cellular machineries (Radonjic et al. 2005; Aragon et al. 2008; Broach 2012; Miles et al. 2013; Kuang et al. 2014; Mews et al. 2014; Guidi et al. 2015; McKnight et al. 2015; Rutledge et al. 2015; Young et al. 2017; Sagot and Laporte 2019a,b; Swygert and Tsukiyama 2019). Quiescence is central to many important biological processes and is conserved from unicellular eukaryotes to multicellular organisms (Cheung and Rando 2013; Sagot and Laporte 2019a). We recently showed that quiescent $(\mathrm{Q})$ cells show a 30-fold drop in mRNA levels and that this massive transcriptional shutoff is dependent on the conserved histone deacetylase (HDAC) Rpd3 (McKnight et al. 2015). Rpd3 is recruited to the majority of gene promoters in Q cells, leading to global hypoacetylation of chromatin and gene repression (McKnight et al. 2015). Further, transcriptional shutdown correlates with loss of Rpb3, one of the subunits of Pol II (McKnight et al. 2015; Young et al. 2017).

To systematically interrogate the chromatin changes at a transcriptionally active locus upon inhibition of transcription, we used Epi-Decoder, a tag-chromatin immunoprecipitation (ChIP)-barcode (BC)-sequencing technology in budding yeast (Korthout et al. 2018). Epi-Decoder enables the decoding of the proteome of a single barcoded genomic locus by DNA sequencing and BC counting. It takes advantage of the power of cellular DNA barcoding (Yan et al. 2008; Fowler and Fields 2014; Vlaming et al. 2016; Chabbert et al. 2018; Kebschull and Zador 2018; Roy et al. 2018) and yeast genetics (Duina et al. 2014) and provides a quantitative approach orthogonal to capture-mass-spectrometry efforts (Schmidtmann et al. 2016; Wierer and Mann 2016; Myers et al. 2018). Here we describe an expanded library of yeast strains carrying a double-BC transcribed reporter gene integrated at the $\mathrm{HO}$ locus (Epi-Decoder-HO). Of this library, we used a dedicated subset of approximately 700 (putative) chromatin proteins (for more details, see Supplemental Materials and Methods) of which the binding at the reporter locus can be assessed in parallel and in triplicate with three independent $\mathrm{BC}$ in a single sample. This Chrom- $3 \times \mathrm{BC}$ library was used to determine the local chromatin-proteome rewiring at the barcoded transcribed locus in response to transcription inhibition by chemical perturbation and during quiescence.

\section{Results}

\section{Generation of improved Epi-Decoder-HO libraries}

To measure local proteome dynamics at a promoter and terminator region upon transcriptional inhibition, we first improved the pre- viously reported Epi-Decoder-HO library to optimize the comparison of multiple time points and conditions. Epi-Decoder is a strategy for decoding the local proteome of a single genomic locus (Korthout et al. 2018). It relies on short ( 16- to 20-bp) DNA BCs integrated at a common locus in the genome. Here we used the constitutively expressed KanMX marker gene integrated at the $\mathrm{HO}$ locus and flanked by two BCs-BC_UP (promoter region) and BC_DN (terminator region)-that are $1.5 \mathrm{~kb}$ apart (HOBarcoders). The barcoded KanMX cassette is a kanamycin gene controlled by the heterologous AgTEF1 promoter and terminator from Ashbya gossypii, a yeast related to Saccharomyces cerevisiae. This 1.5-kb reporter-gene cassette replaces the coding sequence of the $\mathrm{HO}$ gene and is therefore flanked by the endogenous $\mathrm{HO}$ promoter and terminator sequences as well as an origin of replication (ARS404) 53 bp downstream from BC_DN. This reporter locus has previously been used in screens for various chromatin- and transcription-related features (Verzijlbergen et al. 2011; Chen et al. 2013; Vlaming et al. 2016, 2019; Korthout et al. 2018). The library of HO-Barcoders was combined with a genome-wide library of proteins tagged with a common epitope tag, a tandem affinity purification (TAP) tag (Fig. 1A; Supplemental Fig. S1A). Upon pooling, cross-linking, ChIP, amplification of the barcoded regions, and counting the BCs by massive parallel sequencing (Fig. 1B), the abundance of each BC (ChIP/input) reports on the occupancy of each tagged protein at its barcoded locus (Verzijlbergen et al. 2011; Vlaming et al. 2016; Korthout et al. 2018). Integral for high-throughput assessments like this is the ability to multiplex many individual TAP-tag clones (approximately 4800 in total) in the ChIP assays using unique BCs and indexing strategies. Here we expanded the previous set of HO-Barcoders from about 1100 to about 2500 (Douglas et al. 2012), enabling coverage of the full Epi-Decoder library with only two separate subsets (Fig. 1B; Supplemental Fig. S1A). The expanded Barcoder-HO library was combined with the TAP-tag library in three different ways (see Methods) such that every TAP-tag is linked to three independent HO-Barcoders (I, II, and III) and that a triplicate TAP-tag subset of approximately 700 chromatin proteins $($ Chrom $-3 \times \mathrm{BC})$ was created that can be processed as one pool owing to nonoverlapping $\mathrm{BC}$ pairs in the replicates.

\section{Interrogation of protein binding with multiple DNA BCs}

Epi-Decoder uses DNA BC counting as a reporter for protein binding (Korthout et al. 2018). With the three independent EpiDecoder-HO libraries (Fig. 1B), we first assessed to what extent variation in quantification of protein binding is caused by the $\mathrm{BC}$ sequences. The protein-binding patterns in each of the three EpiDecoder-HO libraries strongly correlated between the replicates (Fig. 1C) for both BC_UP and BC_DN (Supplemental Fig. S1B,C; Supplemental Table S1), and this was further confirmed by the analysis of individual clones (see below). Although it is possible that specific BC sequences could cause a bias for detection of certain proteins, in general our results show that the inferred protein-binding scores are largely independent of the short BC sequences. The use of multiple Tag-Barcode combinations further increases the confidence of single-BC measurements. For example, we have previously shown that Ssl2 and Tfa2, two factors known for their roles in transcription initiation, also show high binding at the $\mathrm{HO}$ terminator region (Korthout et al. 2018). By replicating this finding with three independent barcodes, we can now negate the possibility that BC effects caused binding of Ssl2 and Tfa2 at the HO terminator region (Supplemental Table S1). The consistent

\section{Genome Research}

www.genome.org 
A
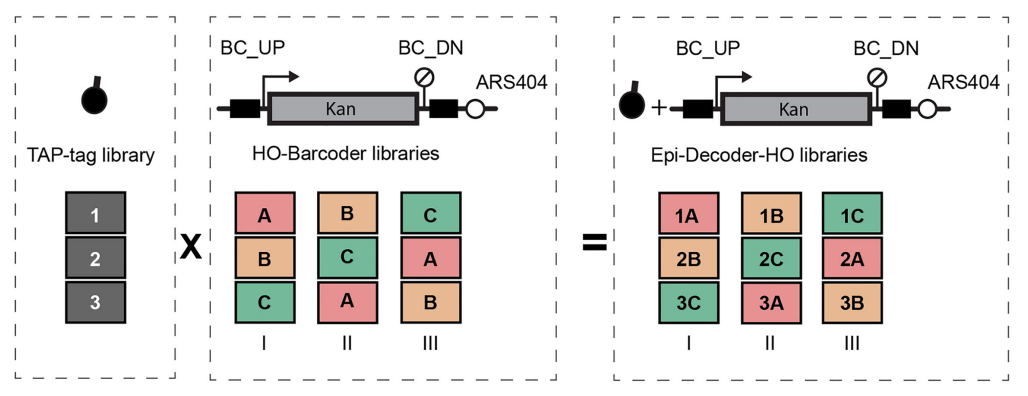

B

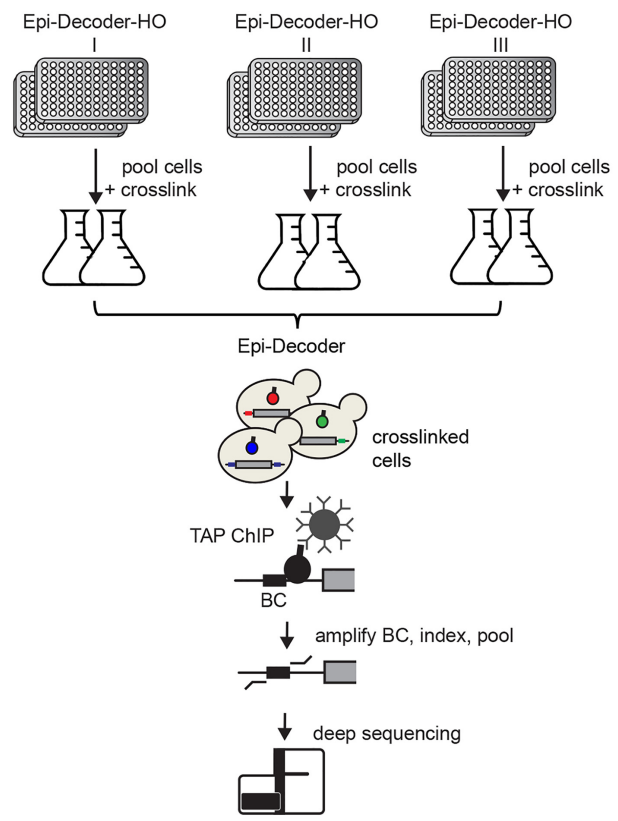

C

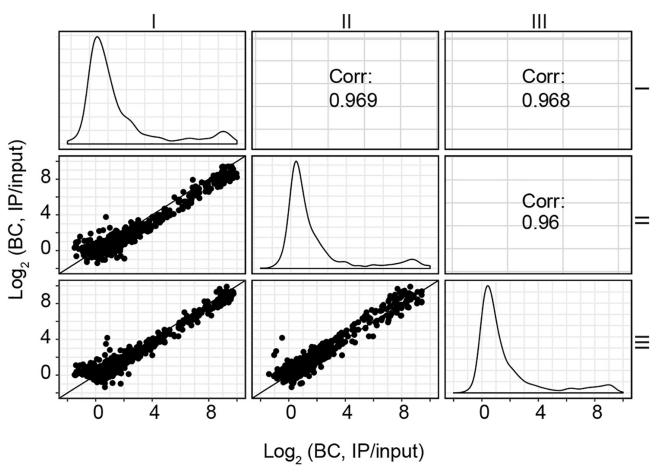

Figure 1. Outline of expanded and optimized Epi-Decoder analysis. (A) Three Epi-Decoder-HO libraries were generated by crossing the TAP-tag library to an expanded HO-BC library in three different ways to combine each TAP-tag protein with three independent barcodes (BCs) (see also Supplemental Fig. S1A). The HO-BC locus consists of a constitutively expressed 1.5-kb KanMX resistance gene integrated at the $\mathrm{HO}$ locus, controlled by the heterologous AgTEF1 promoter and terminator from Ashbya gossypii, and flanked by a promoter-proximal BC_UP and a terminator-proximal BC_DN. Downstream from BC_DN lies an origin of replication (ARS404). (B) Clones of each Epi-Decoder-HO library are combined and processed in two separate pools and used for ChIP of TAP-tagged proteins (spheres with black handle). The $\mathrm{BCs}$ (colored lines), which flank the KanMX reporter gene (gray box) at the $\mathrm{HO}$ locus, are amplified from ChIP and input and indexed, allowing for the pools to be combined and counted by massive parallel sequencing. The relative BC count (IP/input) reports on protein abundance of each TAP-tagged protein (approximately 4250) at the barcoded locus. (C) Comparison of the binding scores (IP/input) of both BC_UP and BC_DN of chromatin binders (as determined previously by Korthout et al. 2018) in the three Epi-Decoder-HO libraries. Indicated are the Spearman's correlation coefficients, and the diagonal line represents $x=y$. Density plots show the distribution of the BC counts in each of the three replicates. For counts of all proteins examined, see Supplemental Table S1. The results for BC_UP and BC_DN separately are shown in Supplemental Figure S1, B and C. and robust binding of Ssl2 and Tfa 2 at the terminator region in the absence of other basal TFs suggests a noncanonical function of these factors.

\section{A chromatin TAP-tag subset library for capturing dynamic local chromatin proteomes}

Having confirmed that Epi-Decoder provides a robust assay to quantitatively measure protein binding at a single locus of thousands of proteins in parallel, we focused on the chromatin TAP-tag subset (Chrom-3 $\times$ BC; see Methods) (Fig. 2A) to determine the dynamics of the local chromatin proteome following chemical inhibition of Pol II. The antifungal agent $\mathrm{PH}$ is a metal ion chelator that sequesters $\mathrm{Zn}^{2+}$ ions, which are essential for the activity of RNA polymerases (Scrutton et al. 1971; Markov et al. 1999; Cramer et al. 2000; McCann et al. 2012). The addition of $\mathrm{PH}$ to cells rapidly affects mRNA levels (Grigull et al. 2004; Miller et al. 2011; Wada and Becskei 2017). AU is an inhibitor of IMP dehydrogenase (IMPDH), the rate-limiting enzyme in de novo GTP synthesis (Grigull et al. 2004; Kaplan et al. 2012; Ljungdahl and Daignan-Fornier 2012). The treatment of cells with $\mathrm{AU}$ results in depletion of intracellular nucleotide pools, thereby affecting transcription elongation (Handschumacher and Welch 1956; Exinger and Lacroute 1992; Shaw and Reines 2000; Shaw et al. 2001; Mason and Struhl 2005). To investigate the consequences of inhibition of Pol II at the chromatin template, the Chrom $-3 \times \mathrm{BC}$ Epi-Decoder library was incubated with $\mathrm{PH}$ and $\mathrm{AU}$, and the local proteomes were determined at several subsequent time points (Fig. 2A). All experiments were performed at low temperature $\left(16^{\circ} \mathrm{C}\right)$ to facilitate the capturing of dynamic binding events. In addition, cells were arrested in G1 to avoid cell-cycle-dependent effects (Supplemental Fig. S2A; O'Duibhir et al. 2014). Independent triplicate measurements in the Chrom- $3 \times \mathrm{BC}$ pool and sample multiplexing enabled efficient analysis of the dynamics of known chromatin proteins across multiple conditions and time points.

We confirmed that $\mathrm{PH}$ and $\mathrm{AU}$ are active under these conditions: Both compounds inhibited cell growth (Supplemental Fig. S2B), and addition of PH led to induction of $Z R T 1$ mRNA whereas addition of $\mathrm{AU}$ led to induction of IMD2 mRNA (Fig. 2B; Supplemental Fig. S2C), 
A

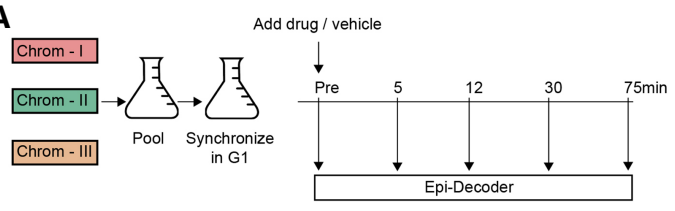

C

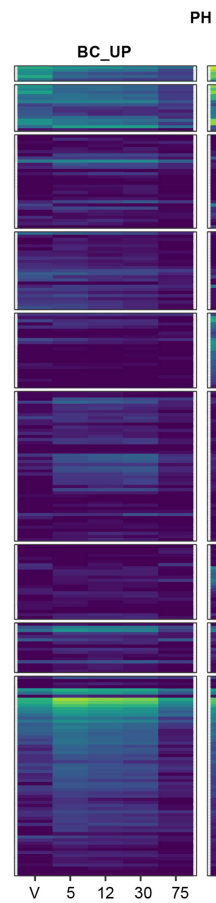

$\mathrm{PH}$
BC_DN

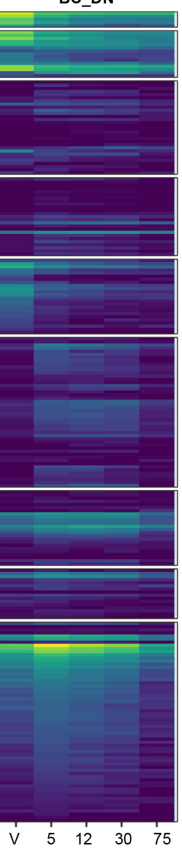

B

AU

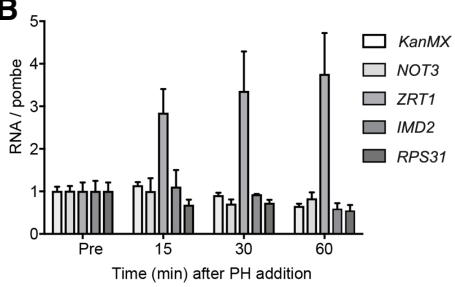

BC_DN
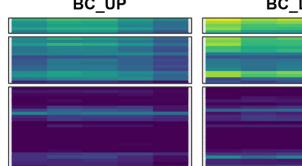

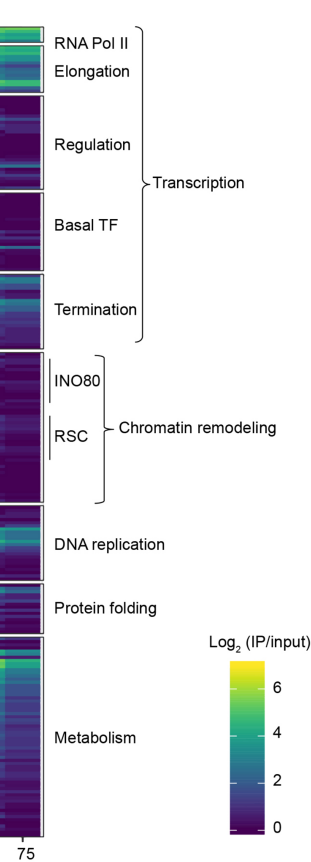

Time $(\mathrm{min})$ after drug addition

Figure 2. Chromatin-proteome dynamics at the Barcoded-HO locus upon chemical inhibition of transcription. $(A)$ Three versions of the chromatin TAP-tag subset, each containing a unique set of $B C$ pairs, were combined into one pool (Chrom $-3 \times B C)$. The culture was incubated with a-factor pheromone to synchronize the cells in G1, after which $\mathrm{PH}$ or $\mathrm{AU}$ was added and samples were collected at the time points indicated for Epi-Decoder analysis, RNA analysis, and flow cytometry. A sample treated with vehicle was collected at the last time point, and a control sample was taken of a G1-arrested culture without treatment. All experiments were performed at $16^{\circ} \mathrm{C}$ to facilitate capturing dynamic binding events. (B) Analysis of mRNA changes over time by RT-qPCR confirmed the decay of most transcripts and an increase in the PH-responsive $Z R T 1$ gene under conditions shown in panel $A$. RNA levels are relative to untreated (Pre) and normalized to a transcript from a spike-in of untreated Schizosaccharomyces pombe cells (see Methods) to correct for global changes (mean of three biological replicates \pm SD). (C) Heatmap of the binding scores (mean IP/input of three biological replicates) of selected proteins with a binding score $>0.5$ at any of the four local proteome time series indicated. For mean binding scores of all proteins examined in the Chrom $-3 \times \mathrm{BC}$ library, see Supplemental Table S2. Proteins were manually clustered and ranked in functional subcategories.

which is in agreement with previous observations (Shaw and Reines 2000; Grigull et al. 2004) that inhibition of transcription by these drugs is not complete. Under these experimental conditions and time points, we observed little decay of mRNA levels of the barcoded KanMX gene or other genes examined (Fig. 2B; Supplemental Fig. S2C), in agreement with the observed temperature effects on RNA decay kinetics (Lotan et al. 2005). In contrast, global inspection of binders at the barcoded KanMX gene showed that the addition of $\mathrm{PH}$ or $\mathrm{AU}$ led to many rapid and pronounced changes at the chromatin level at BC_UP and BC_DN (Fig. 2C). Generally, changes in protein binding were stronger in $\mathrm{PH}$ than in $\mathrm{AU}$, confirming that the observed effects are specific for the treatment.
Rewiring of the core transcription machinery at chromatin upon inhibition of Pol II

The global overview of the dynamic chromatin proteomes (average of three replicates) (Fig. 2C) revealed broad rearrangements, especially of protein complexes related to transcription. Other proteins were unaffected, such as the replication proteins specifically associated with BC_DN proximal to the origin of replication ARS404. This was further illustrated by the analysis of individual replicate measurements of proteins representing different aspects of chromatin biology (Fig. 3A). Because these three replicates were based on different BC pairs, the reproducible results indicate that $\mathrm{BC}$ sequences did not generally affect the protein-binding measurements in Epi-Decoder. As observed previously (Korthout et al. 2018), Pol II and transcription elongation factors were found at BC_UP and were more abundant at BC_DN, initiation factors and basal TFs were more enriched at BC_UP, and transcription termination factors and replication proteins were specifically bound to BC_DN (Figs. 2C, 3A; Supplemental Table S2). To determine the consequences of transcription inhibition for the transcription machinery in more detail, we focused on Pol II and transcription elongation factors. All the Pol II subunits present in our Epi-Decoder library showed a pronounced and progressive loss of binding upon addition of $\mathrm{PH}$ at BC_UP and BC-DN. In AU, Pol II binding was also reduced but to a lesser extent (Fig. 3B). Therefore, we here focused on the changes following treatment with $\mathrm{PH}$. Loss of binding can be caused by lower levels of the protein owing to protein degradation or by redistribution of the protein. To distinguish between these two possibilities, we determined the protein level of Rpo21, the largest subunit of Pol II, by immunoblot analysis and found that treatment with $\mathrm{PH}$ did not lead to reduced Rpo21 protein levels (Fig. 3C; Supplemental Fig. S3A), whereas treatment with AU showed a modest decrease (Fig. 3D; Supplemental Fig. S3B). Therefore, the reduced binding of Pol II subunits in PH was not accompanied by increased Pol II protein degradation.

Finally, the strong reduction in Pol II binding observed in EpiDecoder in PH at the barcoded KanMX gene under the control of the AgTEF1 promoter and terminator could be validated by ChIP-qPCR at the $5^{\prime}$ and $3^{\prime}$ ends of the endogenous TEF1 gene (Fig. 3E; Supplemental Fig S3C). Thus, in addition to the current knowledge that Pol II activity is inhibited directly by PH and indirectly by AU (Scrutton et al. 1971; Markov et al. 1999; Cramer et al. 2000; McCann et al. 2012), our results show that chemical

\section{Genome Research}

www.genome.org 
A

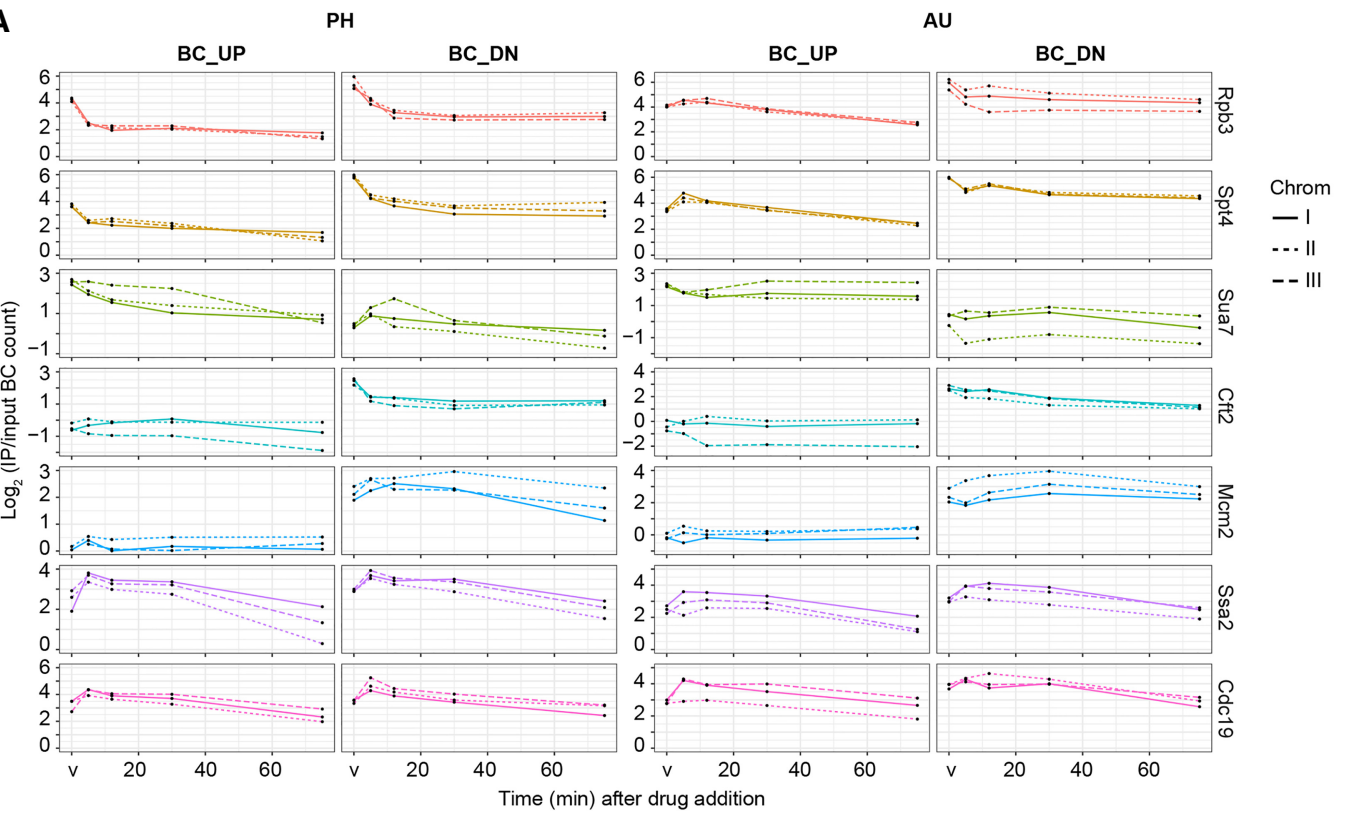

B
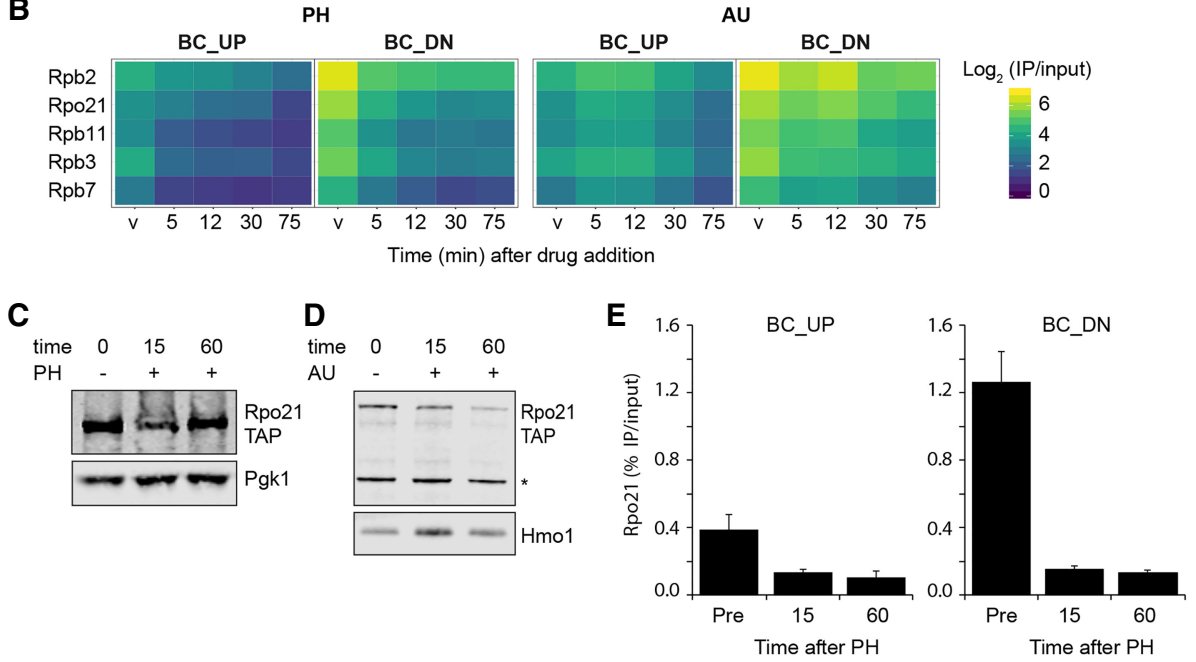

Figure 3. Treatment with phenanthroline $(\mathrm{PH})$ leads to rapid loss of Pol II and transcription-associated proteins. $(A)$ Differential and dynamic binding behavior of proteins representing different chromatin processes upon treatment with PH and AU. The lines indicate the three different BC pairs of the indicated TAP-tagged proteins in the Chrom-3×BC library ( $\log _{2}$ IP/input at time points indicated in Fig. 2). (B) Zoom-in of heatmap of Figure 2 showing the Pol II subunits present in the library. $(C, D)$ Immunoblot analysis of the largest subunit of Pol II (Rpo21-TAP) with and without PH and AU treatment in $\mathrm{G} 1$-arrested cells at $16^{\circ} \mathrm{C}$. Pgk1, Hmo1, and a nonspecific band $\left(^{*}\right)$ were used as loading controls. (E) ChIP-qPCR analysis of Rpo21 binding at the BC_UP and BC_DN regions in G1-arrested cells treated with (15 and $60 \mathrm{~min}$ ) and without (Pre) PH at $16^{\circ} \mathrm{C}$ (average of three biological replicates $\pm S D$ ).

inhibition of Pol II also leads to partial eviction of the core Pol II machinery from the chromatin template.

\section{Epi-Decoder uncovers protein-protein relationships}

Several proteins and protein complexes have been biochemically or genetically linked to the process of transcription elongation (Koerber et al. 2009; Venters and Pugh 2009; Kwak and Lis 2013; Pufall and Kaplan 2013; Cucinotta and Arndt 2016; Van Oss et al. 2017; Chen et al. 2018; Vinayachandran et al. 2018). However, how the interactions between the core transcriptional machinery and elongation factors are dynamically orchestrated on the chromatin template is still incompletely understood
(Mayer et al. 2010; Vinayachandran et al. 2018). Inspection of the dynamic proteomes at the $\mathrm{HO}$ locus showed that elongation factors did not all respond equally to inhibition of Pol II by $\mathrm{PH}$ (Fig. 4A) and AU (Supplemental Fig. S4A). The conserved elongation factors Elf1, Spn1, Spt6, and DSIF (Spt4/5) were rapidly and progressively evicted from chromatin, closely resembling the dynamics of Pol II upon PH treatment (Figs. 3B, 4A). This indicates that most elongation factors depend on Pol II for recruitment and maintenance. The treatment with AU, performed and processed in parallel, showed more modest effects on binding of Pol II and the elongation factors (Supplemental Fig. S4A,B).

The FACT complex, composed of Spt16 and Pob3, is known to be recruited to chromatin by the act of transcription and its 
A

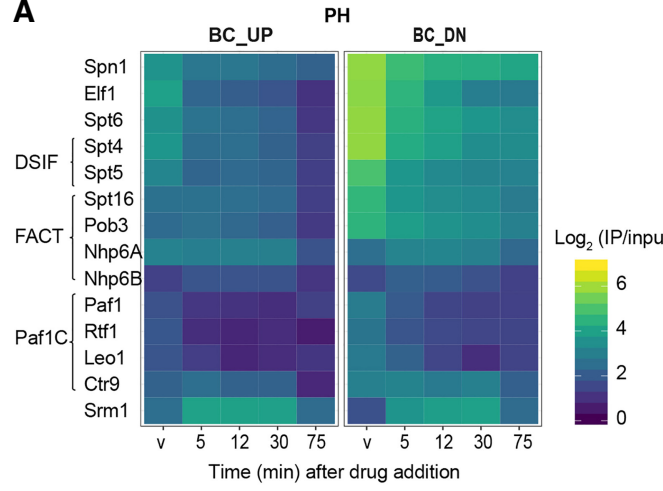

C

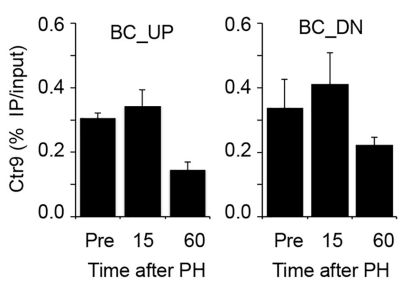

D

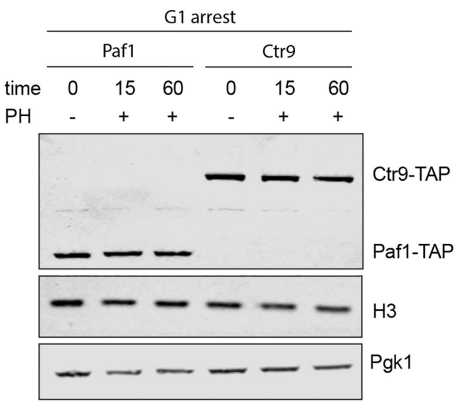

B

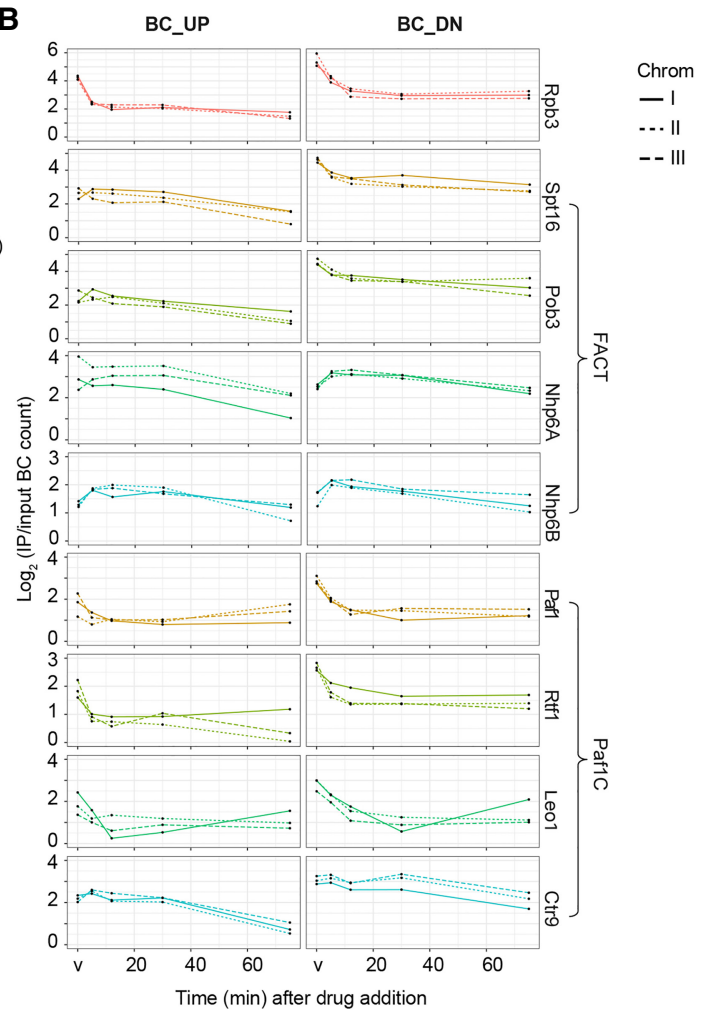

E

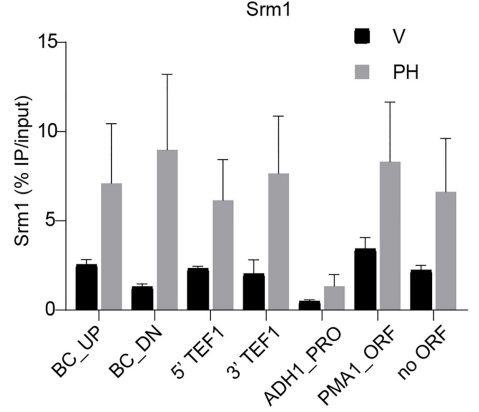

Figure 4. Differential response of transcription elongation factors to chemical inhibition of Pol II. (A) Zoom-in on the heatmap of Figure 2 (PH treatment), showing proteins annotated to transcription elongation. $(B)$ Independent replicates of proteins related to FACT and Paf1C (Log 2 IP/input at time points as in Fig. 2). The lines show the three different $B C$ pairs of the indicated TAP-tagged proteins in the Chrom-3 $\times B C$ library. (C) ChIP-qPCR analysis of Ctr9 binding at the BC UP and BC DN regions in G1-arrested cells treated with $\left(15\right.$ and $60 \mathrm{~min}$ ) and without (Pre) $\mathrm{PH}$ at $16^{\circ} \mathrm{C}$ (average of three biological replicates \pm SD). (D) Immunoblot analysis of Ctr9-TAP and Paf1-TAP with and without PH treatment in G1-arrested cells at $16^{\circ} \mathrm{C}$. Pgk1 and $\mathrm{H} 3$ were used as loading controls. (E) ChIP-qPCR analysis of Srm 1 binding in $\mathrm{G} 1$-arrested cells at $16^{\circ} \mathrm{C}$, treated for 15 min with vehicle $(\mathrm{V})$ or $\mathrm{PH}$ (average of three biological replicates \pm SD). Analyzed loci are the BC_UP and BC_DN regions, the $5^{\prime}$ and $3^{\prime}$ end of the endogenous TEF1 gene, the ADH1 promoter, the PMA1 open reading frame, and a nontranscribed locus (for more details, see Supplemental Table S5; van Welsem et al. 2018).

associating factors Nhp6a and Nhp6a, two high mobility group (HMG) proteins (Ruone et al. 2003; Kwak and Lis 2013; McCullough et al. 2015; Martin et al. 2018; Pathak et al. 2018). At BC_UP, FACT eviction was delayed compared with eviction of Pol II upon PH treatment (Fig. 4A,B), suggesting that FACT binding might depend on more stable factors at transcribed genes such as histones or histone modifications (Martin et al. 2018; Vinayachandran et al. 2018). At BC_DN, the two core FACT subunits Spt16 and Pob3 were rapidly lost upon addition of $\mathrm{PH}$, closely resembling the behavior of Pol II and other elongation factors. In contrast, the HMG proteins Nhp6a and Nhp6b did not show reduced binding after Pol II inhibition (Fig. 4A,B). This difference may reflect the ability of HMG proteins to bind DNA and nucleosomes directly, independently of other chromatin proteins.

Recent structural studies show that Paf1C, DSIF (Spt4/5), and Spt6 can form an intricate protein network around elongation-permissive Pol II (Vos et al. 2018; Xie et al. 2018). Indeed, like DSIF and Spt6, most Paf1C subunits showed reduced binding to chromatin when Pol II was inhibited and evicted by $\mathrm{PH}$ and $\mathrm{AU}$ (Fig. 4A,B; Supplemental Fig S4A,B). However, binding of Ctr9, the largest subunit and a key scaffold protein of Paf1C (Deng et al. 2018; Vos et al. 2018; Xie et al. 2018), was largely unaffected in PH, with a small decrease only observed at later time points (Fig. 4A, B). We confirmed the delayed loss of Ctr9 by ChIP-qPCR analysis of the barcoded promoter and terminator regions (Fig. 4C), as

\section{Genome Research}

www.genome.org 
well as the endogenous TEF1 locus (Supplemental Fig. S4C). The differences in kinetics were not caused by differences in protein abundance, because Ctr9 showed very similar global expression levels as the Paf1 and Pol II subunits (Supplemental Fig. S4D). We also tested whether the delayed loss of Ctr9 was caused by a difference in crosslinking sensitivity (Zaidi et al. 2017; De Jonge et al. 2019). However, Paf1 and Rpo21 were not more sensitive to shorter cross-linking conditions than was Ctr9 (Supplemental Fig. S4E). Finally, addition of PH did not lead to major changes in the expression level of Rpo21 and Paf1, compared with Ctr9, the latter remaining bound when Rpo21 and Paf1 had already been evicted upon PH treatment (Fig. 4D). Together, our results show that binding of Ctr9 at transcribed chromatin is independent of Pol II and Paf1C subunits, suggesting that Ctr9 has a transcription elongation-independent mechanism to bind to DNA, perhaps mediated by its known interactions with DNA and nucleosomes (Musso et al. 2000; Deng et al. 2018; Vos et al. 2018). These binding activities may be responsible for anchoring Ctr9 to PH-perturbed chromatin in the absence of its complex partners and Pol II. Of note, under conditions of transcriptional repression in Q cells, Ctr9 followed the pattern of the other Paf1C members (see below), showing that its dynamics are context dependent.

In addition to uncovering protein-binding dynamics within well-known protein complexes, the parallel analysis of hundreds of proteins also offers the possibility to obtain more insight into proteins of which the chromatin functions have been less well annotated. Here we more closely examined Srm1 (also known as Prp20), a homolog of human Regulator of Chromosome Condensation 1 (RCC1). Srm1/RCC1 is a guanine nucleotide exchange factor that localizes to the nucleus, binds nucleosomes in a dynamic way, and occupies a large part of the yeast genome (Nemergut et al. 2001; Koerber et al. 2009; Makde et al. 2010; Wu et al. 2011; Bierbaum and Bastiaens 2013; McGinty and Tan 2016). In the Epi-Decoder analysis, all proteins are examined in one pool and with the same tag, thereby delivering qualitative as well as relative quantitative information. The high BC counts observed for Srm 1 show that it binds efficiently to chromatin, in the same range as the abundant transcription elongation factors mentioned above (Fig. 4A; Supplemental Fig. S4A). Analysis of Srm 1 by ChIP-qPCR confirmed the efficient binding of this protein and an increase after PH treatment at the reporter locus as well as endogenous loci, including a nontranscribed region (Fig. 4E). This, together with the genome-wide binding pattern (Koerber et al. 2009), indicates that Srm1 should be considered as a common component of yeast chromosomes. However, the distribution and kinetic behavior of Srm 1 did not mirror that of the transcription elongation factors: Upon treatment with $\mathrm{PH}$, Srm1 binding increased (Fig. 4A,E), whereas binding of Pol II and most elongation proteins decreased. Although not quantitatively the same, the behavior of Srm 1 is more similar to that of the chromatin remodelers INO80 and RSC, several metabolic enzymes (Fig. 2C), the heat shock protein Ssa2 (Fig. 3A; Supplemental Fig. S4F), and HMG protein Hmo1 (Supplemental Fig. S4F). In contrast, the HMG-like protein Spt2, a chaperone involved in histone recycling over transcribed regions of active genes (Nourani et al. 2006; Chen et al. 2015), was decreased in active regions (Supplemental Fig. S4F). The dynamic behavior of Srm1, Ssa2, Hmo1, and Spt2 after $\mathrm{PH}$ treatment was not caused by changes in global protein levels (Supplemental Fig. S4G). Together, these results suggest that Srm1 is an abundant chromatin protein with the potential to affect chromatin structure and function but that it might not act as a canonical transcription elongation factor.
Rearrangement of the chromatin proteome of the barcoded $\mathrm{HO}$ locus upon entry in quiescence

Finally, we investigated how the chromatin proteome of the barcoded $\mathrm{HO}$ locus was altered by the strong physiological transcriptional shut down during quiescence. Chrom- $3 \times \mathrm{BC}$ Epi-Decoder library pools were grown in YEPD for $7 \mathrm{~d}$ at $30^{\circ} \mathrm{C}$, which causes cells to arrest in saturation with G1 DNA content. Stationary phase cultures consist of two populations: Q cells, which are more uniform, long-lived, and stress resistant, and nonquiescent (NQ) cells, which are more heterogeneous, short-lived, and stress sensitive (Allen et al. 2006; Aragon et al. 2008; Li et al. 2013; Young et al. 2017; Sagot and Laporte 2019a,b). NQ cells were also isolated and processed as a reference.

Analysis of the RNA Pol II machinery, transcription elongation factors, basal TFs, and transcription termination and RNA processing factors showed a pronounced loss of the core transcription machinery in Q and NQ cells (Fig. 5A,B; Supplemental Fig. S5A-E; Supplemental Table S2). Overall, the magnitude of the changes was higher than that observed in the G1 cells treated with PH with several exceptions. The FACT-associating factors Nhp6a and Nhp6a showed increased chromatin binding in Q cells (Fig. 5B; Supplemental Fig. S5A), in contrast to PH-treated cells (Fig. 4; Supplemental Fig. S4). Tho1, a protein associated with transcribed chromatin, showed increased abundance in $\mathrm{Q}$ and NQ cells but not in PH (Fig. 5B; Supplemental Fig. S5A), suggesting that Tho1 might have $\mathrm{Q} / \mathrm{NQ}$ cell-specific functions and that its recruitment is independent of ongoing transcription, in contrast to canonical transcription elongation proteins.

To gain more insight into the transcriptional repression of the barcoded $H O$ locus, we inspected transcriptional regulators at the BC_UP promoter region (Fig. 5C; Supplemental Fig. S5B). Q cells showed binding of the quiescence-specific transcription repressor $\mathrm{Xbp} 1$, which was absent from mid-log cells and during $\mathrm{PH}$ treatment. This protein is known to recruit the HDAC Rpd3, mediating the global shutdown of gene expression in Q cells (McKnight et al. 2015). Indeed, Rpd3 and members of the Rpd3L complex were mostly undetectable in mid-log but readily detected in $\mathrm{Q}$ cells. Members unique for the Rpd3S complex were not detected (Supplemental Table S3). This well-established repression pathway was accompanied by increased abundance of several TFs such as Mcm1, Mbf1, Tbf1, Abf1, and Swi6 (Fig. 5C; Supplemental Fig. S5B).

Besides the core transcriptional machinery, TFs, and Rpd3L, we observed several other changes in the chromatin proteomes (Fig. 5C-E; Supplemental Fig. S5B-G). Yeast Histone H1 (Hho1) showed increased abundance in $\mathrm{Q}$ and NQ cells (Fig. 5C; Supplemental Fig. S5B). HMG protein 1 (Hmo1), which has been shown to function as a linker histone and promote chromatin compaction (Panday and Grove 2016), showed the same trend (Fig. 5C; Supplemental Fig. S5B). In contrast, the negative regulator of transcription Spt2 left the chromatin in Q and NQ cells, as it did in PH-treated cells. These changes were paralleled by the recruitment of the ATP-dependent chromatin remodeling complexes INO80 and RSC, as well as several heat shock proteins and other factors involved in protein folding (Fig. 5E; Supplemental Fig. $\mathrm{S} 5 \mathrm{C}, \mathrm{F})$. In addition, changes were seen in the binding of metabolic enzymes (Supplemental Fig. S5G). Future functional studies will be required to determine the role of these dynamic interactions in the global transcriptional repression occurring in Q cells.

Finally, quiescence also affected the organization of replication proteins at the origin of replication (ARS404) proximal to 
A
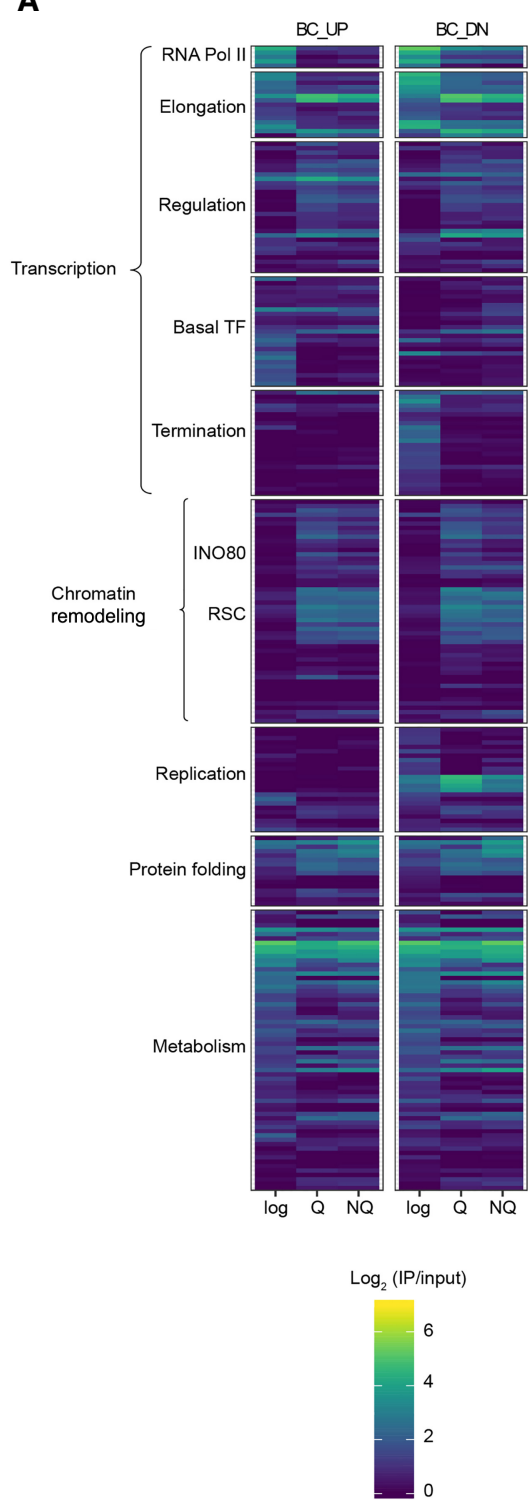

B

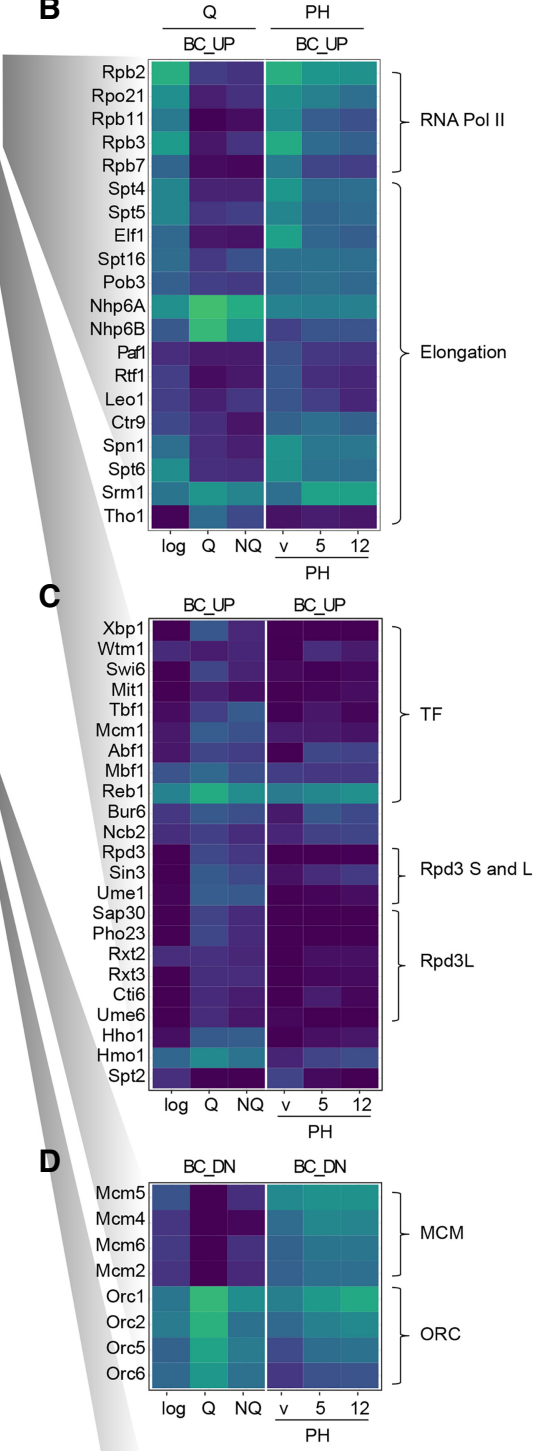

E

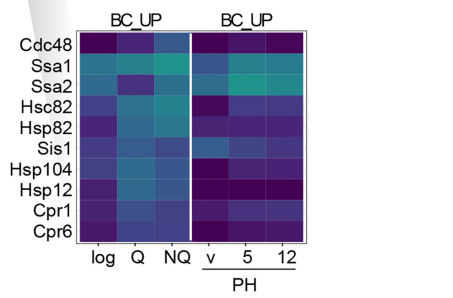

Figure 5. Chromatin-proteome rewiring upon transcriptional repression in quiescence. $(A)$ Heatmap of the HO Epi-Decoder binding scores in mid-log, Q, and NQ cells (mean IP/input of three biological replicates). For mean binding scores of all proteins examined in the Chrom-3×BC library, see Supplemental Table S3. Proteins were manually clustered and ranked in functional subcategories as in Figure 2C. $(B-E)$ Zoom-in of heatmap of panel $A$ (promoter region BC_UP, except replication for which terminator BC_DN next to the origin of replication was used) showing proteins in the indicated annotated clusters. PH treatment during $\mathrm{G} 1$ arrest (vehicle, 5 and $12 \mathrm{~min}$ ) is shown for comparison.

BC_DN (Fig. 5D). In contrast to PH treatment, cells in quiescence showed a complete loss of the minichromosome maintenance complex (MCM), the replicative helicase. Q cells are arrested in a G1-like state, but binding of MCM was maintained in cells arrested in G1 by mating pheromone (Fig. 5D; Liang and Stillman 1997). The loss of MCM was accompanied by increased abundance of

the members of the origin-recognition complex (ORC) (Fig. 5D). This altered balance is consistent with a competition model that has been proposed for the MCM and ORC (Aparicio et al. 1997). Replication origin licensing, which involves the binding of ORC and subsequently the assembly of an inactive form of the replicative helicase MCM, occurs during late mitosis and the G1 phase of the cell cycle. After formation of a replication complex, during $\mathrm{S}$ phase the helicase is activated and the replication initiates (Siddiqui et al. 2013; Bell and Labib 2016). It is possible that clearing MCM from origins in Q cells is important for eliminating any inappropriate activation of replication origins during the prolonged state of arrest. The mechanism causing the absence of MCM and the role thereof in Q-cell biology will require further study, but it should be noted that similar dynamics have been observed in the mouse and Schizosaccharomyces pombe (Madine et al. 2000; Sun et al. 2000; Namdar and Kearsey 2006). Our results suggest that replication origin licensing must be re-established when cells re-enter the cell cycle.

\section{Discussion}

Determining the changes to chromatin composition in response to cell signaling or stress can reveal insights into common mechanisms of gene regulation. Obtaining a comprehensive understanding of chromatin changes, although highly informative (Kim et al. 2010; Weiner et al. 2012; Vinayachandran et al. 2018), is often laborious and therefore not applicable to multiple conditions. Epi-Decoder provides a strategy for identifying and quantifying in an unbiased and systematic manner the proteome of an individual genomic locus by DNA sequencing. Here we used a dedicated comprehensive chromatin library (Chrom- $3 \times \mathrm{BC}$ ) to determine the changes in local chromatin proteomes upon inhibition of Pol II by chemical means or by physiological signals during quiescence.

$\mathrm{PH}$ is a potent inhibitor of Pol II and has fungistatic activity against a broad range of pathogenic fungi and bacteria (McCann et al. 2012). PH and thiolutin, which acts in a similar way, are often used to determine the consequences of perturbation of transcription in yeast. However, the consequences at the chromatin level have not been well characterized even though RNA processing, RNA export, and other downstream processes are linked to transcription and are influenced by chromatin modifying factors. Inhibition of Pol II with $\mathrm{PH}$

\section{Genome Research}

www.genome.org 
resulted in rapid remodeling of the local chromatin proteomes at the $\mathrm{HO}$ locus (Fig. 2C). Pol II was evicted, probably reflecting a structural rearrangement caused by $\mathrm{PH}$. This idea is in agreement with observations that mutants of Pol II with altered catalytic activity show reduced Pol II occupancy at genes (Malik et al. 2017). We did not observe changes in overall abundance of the evicted proteins Rpo21 and Paf1 and several other proteins, indicating that the factors evicted in $\mathrm{PH}$ were not subject to degradation. Besides inhibiting Pol II, PH-a metal chelator-is known to also affect the activity of metalloproteases, including a proteasomeassociated deubiquitinating enzyme (Verma et al. 2002; McCann et al. 2012). Therefore, the stability of the proteins evicted by PH may at least in part be explained by inhibition of proteasome-mediated degradation. The nature and scale of the changes observed after PH treatment, particularly the loss of the core transcription machinery, suggests that inhibition of Pol II can have widespread effects on many nuclear processes in the cell. This should be considered when interpreting downstream effects of chemical inhibitors of transcription, such as mRNA processing and stability.

During quiescence, global changes in histone modifications have been observed (Mews et al. 2014; Young et al. 2017), as well as global repression by Rpd3 (McKnight et al. 2015), chromatin compaction by condensin (Swygert et al. 2019), and reduced Pol II binding (Young et al. 2017). However, the extent to which the epigenome of Q cells is remodeled to support this important developmental state and to allow re-entry into vegetative growth remains poorly understood (Swygert and Tsukiyama 2019). Application of Epi-Decoder to the barcoded $\mathrm{HO}$ locus revealed that upon loss of transcription during quiescence, the chromatin proteome of a transcribed region undergoes a major rewiring that affects transcriptional proteins and replication complexes. In addition, we observed the recruitment of structural proteins, chromatin remodelers, and protein-folding machineries, as well as changes in metabolic enzymes. Although many changes between mid-log and Q cells were also observed upon PH treatment, we observed Q-cell-specific changes as well, including increased occupancy of other transcription-related proteins (Fig. 5C), Hho1, changes in specific metabolic enzymes (e.g., Tdh1, Arg1; Supplemental Fig. S5E), and differential binding of several heat shock proteins (e.g., Ssa1 versus its paralog Ssa2) (Fig. 5E; Supplemental Fig. S5E). We note that some of the observed dynamics in $\mathrm{Q}$ and NQ cells might, at least in part, be driven by altered expression of the proteins in the different cell states. Determining the functional roles of these Q-cell-specific and NQ-cell-specific changes will require further mechanistic studies.

Although Q cells and NQ cells showed overlap in the changes compared with mid-log cells, we also observed important differences, in agreement with the different fates of the long-lived $Q$ and short-lived NQ cells (Li et al. 2013) and the differences in global histone modifications (Young et al. 2017). NQ cells lacked efficient recruitment of $\mathrm{Xbp} 1$, which correlated with lower levels of Rpd3L and INO80. In addition, the loss of the MCM complex was incomplete in NQ cells. Together these changes may contribute to the poor fitness of these cells. Furthermore, the binding of Cdc48 at the BC_UP promoter region and BC_DN terminator region in NQ cells is a possible indicator of the general short-lived nature of NQ cells (Fig. 5E). Cdc48, known as VCP in humans, is an essential and conserved AAA+ ATPase that functions as an unfoldase or segregase, facilitating the extraction of proteins from macromolecular complexes, including chromatin, to enable subsequent degradation by the proteasome (Dantuma et al. 2014; Franz et al. 2016). The recruitment of protein quality-control fac- tors to the chromatin agrees with recent observations that effective and diverse protein quality-control mechanisms are active in the nucleus (Jones and Gardner 2016; Prasad et al. 2018; Frottin et al. 2019; Jones et al. 2019).

Our results show that Epi-Decoder provides a powerful strategy for capturing the temporal binding dynamics of chromatin proteins under varying conditions. We expect that future studies on local chromatin-proteome maps under different conditions and at other genomic loci will offer powerful resources for detailed molecular and functional annotation of chromatin proteins and their interactions and relationships at the genome.

\section{Methods}

\section{Yeast strains and libraries}

Yeast strains used in this study are listed in Supplemental Table S4. Library manipulations on solid media were performed using synthetic genetic array (SGA) technology (Tong and Boone 2006) and a ROTOR instrument (Singer Instruments). Yeast media were prepared as previously described (Tong and Boone 2006; Korthout et al. 2018). Details of library construction and growth conditions are provided in the Supplemental Materials and Methods.

\section{RNA isolation and reverse transcription}

RNA was isolated using the RNeasy mini kit (Qiagen) using the protocol for yeast cells, with a few modifications, essentially as previously described (Korthout et al. 2018) and with a spike-in reference (A8545; a gift from R. Allshire) as described in the Supplemental Materials and Methods. RT-qPCR was performed with the primers described in Supplemental Table S5. Each sample was measured in two technical duplicates, and the average value was taken when combining biological replicates.

\section{Epi-Decoder}

Epi-Decoder was performed and analyzed essentially as described previously (Korthout et al. 2018). Protein binding at BC_UP and BC_DN was analyzed separately with specific primers (Supplemental Table S5). Libraries of the PCR products were mixed in an equimolar fashion and sequenced (single read, $>50 \mathrm{bp}$ ) on a HiSeq 2500/MiSeq platform (Illumina), using one or a mix of custom sequencing primers (Supplemental Table S5). Details of the EpiDecoder protocol and analysis and the $\mathrm{BC}$ counting can be found in the Supplemental Materials and Methods.

\section{ChIP-qPCR}

ChIP-qPCR experiments were performed with IgG Sepharose 6 fast flow beads or epoxy-activated Dynabeads as described previously (Korthout et al. 2018; Vlaming et al. 2019) and in the Supplemental Materials and Methods. Each ChIP was performed in triplicate.

\section{Protein detection by immunoblot and antibodies}

For immunoblotting, strains were grown to mid-log phase or arrested in G1 and processed as described previously (Korthout et al. 2018; Vlaming et al. 2019). Antibodies used and detailed protocols can be found in the Supplemental Materials and Methods. 


\section{Data access}

All raw sequencing data generated in this study and corresponding reference tables (Supplemental Tables S6-S11) have been submitted to the NCBI BioProject database (https://www.ncbi.nlm.nih .gov/bioproject/) under accession number PRJNA610036, and to the NCBI BioSample database (https://www.ncbi.nlm.nih .gov/biosample/) under accession numbers SAMN14271070, SAMN14271071, and SAMN14271072. All processed data are within the paper and the Supplemental Material.

\section{Competing interest statement}

The authors declare no competing interests.

\section{Acknowledgments}

We thank Brenda J. Andrews and Charles Boone for kindly providing the expanded yeast barcoder collection. We thank the RHPC facility of the Netherlands Cancer Institute for providing computational resources, the NKI Genome Core Facility for barcode-sequencing, and Ben Morris of the NKI high-throughput center for help with rearraying yeast libraries by robotics. We thank Craig D. Kaplan, Thom Molenaar, Eliza Mari KwesiMaliepaard, Bas van Steensel, Tineke Lenstra, Marlize van Breugel, and Anne Kappert for insightful discussions and valuable suggestions. F.v.L. was supported by the Dutch Research Council (NWO-VICI-016.130.627). T.T. was supported by the National Institutes of Health (R01 GM111428). C.E.C. was supported by the National Cancer Institute (T32CA009657) and National Institutes of Health (F32GM131554).

Author contributions: D.W.P.-L., T.K., and F.v.L. performed the conception and design, acquisition of data, analysis and interpretation of data, and drafting and revising the article. C.E.C. and T.T. peformed Q-cell library preparation. D.W.P.-L. peformed methodology and validation. T.v.W. peformed acquisition, analysis, and interpretation of data. I.v.K. peformed data analysis. D.E.A. and H.O. peformed the generation of reagents.

\section{References}

Adams CC, Gross DS. 1991. The yeast heat shock response is induced by conversion of cells to spheroplasts and by potent transcriptional inhibitors. J Bacteriol 173: 7429-7435. doi:10.1128/JB.173.23.7429-7435 .1991

Allen C, Büttner S, Aragon AD, Thomas JA, Meirelles O, Jaetao JE, Benn D, Ruby SW, Veenhuis M, Madeo F, et al. 2006. Isolation of quiescent and nonquiescent cells from yeast stationary-phase cultures. J Cell Biol 174: 89-100. doi: $10.1083 /$ jcb.200604072

Aparicio OM, Weinstein DM, Bell SP. 1997. Components and dynamics of DNA replication complexes in S. cerevisiae: redistribution of MCM proteins and Cdc45p during S phase. Cell 91: 59-69. doi:10.1016/S00928674(01)80009-X

Aragon AD, Rodriguez AL, Meirelles O, Roy S, Davidson GS, Tapia PH, Allen C, Joe R, Benn D, Werner-Washburne M. 2008. Characterization of differentiated quiescent and nonquiescent cells in yeast stationary-phase cultures. Mol Biol Cell 19: 1271-1280. doi:10.1091/mbc.e07-07-0666

Bell SP, Labib K. 2016. Chromosome duplication in Saccharomyces cerevisiae. Genetics 203: 1027-1067. doi:10.1534/genetics.115.186452

Bensaude O. 2011. Inhibiting eukaryotic transcription: Which compound to choose? How to evaluate its activity? Transcr 2: 103-108. doi:10 $.4161 /$ trns.2.3.16172

Bierbaum M, Bastiaens PI. 2013. Cell cycle-dependent binding modes of the ran exchange factor RCC1 to chromatin. Biophys J 104: 1642-1651. doi:10.1016/j.bpj.2013.03.024

Broach JR. 2012. Nutritional control of growth and development in yeast. Genetics 192: 73-105. doi:10.1534/genetics.111.135731

Chabbert CD, Adjalley SH, Steinmetz LM, Pelechano V. 2018. Multiplexed ChIP-Seq using direct nucleosome barcoding: a tool for high-through- put chromatin analysis. Methods Mol Biol 1689: 177-194. doi:10 .1007/978-1-4939-7380-4_16

Chen M, Licon K, Otsuka R, Pillus L, Ideker T. 2013. Decoupling epigenetic and genetic effects through systematic analysis of gene position. Cell Rep 3: 128-137. doi:10.1016/j.celrep.2012.12.003

Chen S, Rufiange A, Huang H, Rajashankar KR, Nourani A, Patel DJ. 2015. Structure-function studies of histone $\mathrm{H} 3 / \mathrm{H} 4$ tetramer maintenance during transcription by chaperone Spt2. Genes Dev 29: 1326-1340. doi:10 $.1101 / \operatorname{gad} .261115 .115$

Chen FX, Smith ER, Shilatifard A. 2018. Born to run: control of transcription elongation by RNA polymerase II. Nat Rev Mol Cell Biol 19: 464-478. doi:10.1038/s41580-018-0010-5

Cheung TH, Rando TA. 2013. Molecular regulation of stem cell quiescence. Nat Rev Mol Cell Biol 14: 329-340. doi:10.1038/nrm3591

Cramer P, Bushnell DA, Fu J, Gnatt AL, Maier-Davis B, Thompson NE, Burgess RR, Edwards AM, David PR, Kornberg RD. 2000. Architecture of RNA polymerase II and implications for the transcription mechanism. Science 288: 640-649. doi:10.1126/science.288.5466.640

Cucinotta CE, Arndt KM. 2016. SnapShot: transcription elongation. Cell 166: 1058-1058.e1. doi:10.1016/j.cell.2016.07.039

Dantuma NP, Acs K, Luijsterburg MS. 2014. Should I stay or should I go: $\mathrm{VCP} / \mathrm{p} 97$-mediated chromatin extraction in the DNA damage response. Exp Cell Res 329: 9-17. doi:10.1016/j.yexcr.2014.08.025

De Jonge WJ, Brok M, Kemmeren P, Holstege FCP. 2019. An extensively optimized chromatin immunoprecipitation protocol for quantitatively comparable and robust results. bioRxiv doi.org/10.1101/835926

Deng P, Zhou Y, Jiang J, Li H, Tian W, Cao Y, Qin Y, Kim J, Roeder RG, Patel DJ, et al. 2018. Transcriptional elongation factor Paf1 core complex adopts a spirally wrapped solenoidal topology. Proc Natl Acad Sci 115: 9998-10003. doi:10.1073/pnas.1812256115

Douglas AC, Smith AM, Sharifpoor S, Yan Z, Durbic T, Heisler LE, Lee AY, Ryan O, Göttert H, Surendra A, et al. 2012. Functional analysis with a barcoder yeast gene overexpression system. G3 (Bethesda) 2: 12791289. doi:10.1534/g3.112.003400

Duina AA, Miller ME, Keeney JB. 2014. Budding yeast for budding geneticists: a primer on the Saccharomyces cerevisiae model system. Genetics 197: 33-48. doi:10.1534/genetics.114.163188

Ehara H, Sekine SI. 2018. Architecture of the RNA polymerase II elongation complex: new insights into Spt4/5 and Elf1. Transcr 9: 286-291. doi:10 $.1080 / 21541264.2018 .1454817$

Exinger F, Lacroute F. 1992. 6-Azauracil inhibition of GTP biosynthesis in Saccharomyces cerevisiae. Curr Genet 22: 9-11. doi:10.1007/BF00351735

Feng Y, Zhang Y, Ebright RH. 2016. Structural basis of transcription activation. Science 352: 1330-1333. doi:10.1126/science.aaf4417

Fowler DM, Fields S. 2014. Deep mutational scanning: a new style of protein science. Nat Methods 11: 801-807. doi:10.1038/nmeth.3027

Franz A, Ackermann L, Hoppe T. 2016. Ring of change: CDC48/p97 drives protein dynamics at chromatin. Front Genet 7: 73. doi:10.3389/fgene .2016 .00073

Frottin F, Schueder F, Tiwary S, Gupta R, Körner R, Schlichthaerle T, Cox J, Jungmann R, Hartl FU, Hipp MS. 2019. The nucleolus functions as a phase-separated protein quality control compartment. Science 365: 342-347. doi:10.1126/science.aaw9157

Grigull J, Mnaimneh S, Pootoolal J, Robinson MD, Hughes TR. 2004. Genome-wide analysis of mRNA stability using transcription inhibitors and microarrays reveals posttranscriptional control of ribosome biogenesis factors. Mol Cell Biol 24: 5534-5547. doi:10.1128/MCB.24.12.55345547.2004

Guidi M, Ruault M, Marbouty M, Loïodice I, Cournac A, Billaudeau C, Hocher A, Mozziconacci J, Koszul R, Taddei A. 2015. Spatial reorganization of telomeres in long-lived quiescent cells. Genome Biol 16: 206. doi:10.1186/s13059-015-0766-2

Handschumacher RE, Welch AD. 1956. Microbial studies of 6-azauracil, an antagonist of uracil. Cancer Res 16(10 Part 1): 965-969.

Hantsche M, Cramer P. 2017. Conserved RNA polymerase II initiation complex structure. Curr Opin Struct Biol 47: 17-22. doi:10.1016/j.sbi.2017.03 013

Hsieh TH, Weiner A, Lajoie B, Dekker J, Friedman N, Rando OJ. 2015. Mapping nucleosome resolution chromosome folding in yeast by Micro-C. Cell 162: 108-119. doi:10.1016/j.cell.2015.05.048

Jones RD, Gardner RG. 2016. Protein quality control in the nucleus. Curr Opin Cell Biol 40: 81-89. doi:10.1016/j.ceb.2016.03.002

Jones RD, Enam C, Ibarra R, Borror HR, Mostoller KE, Fredrickson EK, Lin J, Chuang E, March Z, Shorter J, et al. 2019. The extent of Ssa1/Ssa2 Hsp70 chaperone involvement in nuclear protein quality control degradation varies with the substrate. Mol Biol Cell 31: 221-233. doi:10.1091/mbc E18-02-0121

Kaplan CD, Jin H, Zhang IL, Belyanin A. 2012. Dissection of Pol II trigger loop function and Pol II activity-dependent control of start site selection in vivo. PLoS Genet 8: e1002627. doi:10.1371/journal.pgen .1002627

\section{Genome Research}

www.genome.org 
Kebschull JM, Zador AM. 2018. Cellular barcoding: lineage tracing, screening and beyond. Nat Methods 15: 871-879. doi:10.1038/s41592-0180185-X

Kim TS, Liu CL, Yassour M, Holik J, Friedman N, Buratowski S, Rando OJ. 2010. RNA polymerase mapping during stress responses reveals widespread nonproductive transcription in yeast. Genome Biol 11: R75. doi:10.1186/gb-2010-11-7-r75

Koerber RT, Rhee HS, Jiang C, Pugh BF. 2009. Interaction of transcriptional regulators with specific nucleosomes across the Saccharomyces genome. Mol Cell 35: 889-902. doi:10.1016/j.molcel.2009.09.011

Korthout T, Poramba-Liyanage DW, van Kruijsbergen I, Verzijlbergen KF, van Gemert FPA, van Welsem T, van Leeuwen F. 2018. Decoding the chromatin proteome of a single genomic locus by DNA sequencing. PLoS Biol 16: e2005542. doi:10.1371/journal.pbio.2005542

Kuang Z, Cai L, Zhang X, Ji H, Tu BP, Boeke JD. 2014. High-temporal-resolution view of transcription and chromatin states across distinct metabolic states in budding yeast. Nat Struct Mol Biol 21: 854-863. doi:10 $.1038 /$ nsmb. 2881

Kwak H, Lis JT. 2013. Control of transcriptional elongation. Annu Rev Genet 47: 483-508. doi:10.1146/annurev-genet-110711-155440

Lauinger L, Li J, Shostak A, Cemel IA, Ha N, Zhang Y, Merkl PE, Obermeyer S, Stankovic-Valentin N, Schafmeier T, et al. 2017. Thiolutin is a zinc chelator that inhibits the Rpn11 and other JAMM metalloproteases. Nat Chem Biol 13: 709-714. doi:10.1038/nchembio.2370

Li L, Miles S, Melville Z, Prasad A, Bradley G, Breeden LL. 2013. Key events during the transition from rapid growth to quiescence in budding yeast require posttranscriptional regulators. Mol Biol Cell 24: 3697-3709. doi:10.1091/mbc.e13-05-0241

Li X, Egervari G, Wang Y, Berger SL, Lu Z. 2018. Regulation of chromatin and gene expression by metabolic enzymes and metabolites. Nat Rev Mol Cell Biol 19: 563-578. doi:10.1038/s41580-018-0029-7

Liang C, Stillman B. 1997. Persistent initiation of DNA replication and chromatin-bound MCM proteins during the cell cycle in cdc6 mutants. Genes Dev 11: 3375-3386. doi:10.1101/gad.11.24.3375

Ljungdahl PO, Daignan-Fornier B. 2012. Regulation of amino acid, nucleotide, and phosphate metabolism in Saccharomyces cerevisiae. Genetics 190: 885-929. doi:10.1534/genetics.111.133306

Lotan R, Bar-On VG, Harel-Sharvit L, Duek L, Melamed D, Choder M. 2005. The RNA polymerase II subunit Rpb4p mediates decay of a specific class of mRNAs. Genes Dev 19: 3004-3016. doi:10.1101/gad.353205

Madine MA, Swietlik M, Pelizon C, Romanowski P, Mills AD, Laskey RA. 2000. The roles of the MCM, ORC, and Cdc6 proteins in determining the replication competence of chromatin in quiescent cells. J Struct Biol 129: 198-210. doi:10.1006/jsbi.2000.4218

Makde RD, England JR, Yennawar HP, Tan S. 2010. Structure of RCC1 chromatin factor bound to the nucleosome core particle. Nature 467: 562566. doi:10.1038/nature09321

Malik I, Qiu C, Snavely T, Kaplan CD. 2017. Wide-ranging and unexpected consequences of altered Pol II catalytic activity in vivo. Nucleic Acids Res 45: 4431-4451. doi:10.1093/nar/gkx037

Markov D, Naryshkina T, Mustaev A, Severinov K. 1999. A zinc-binding site in the largest subunit of DNA-dependent RNA polymerase is involved in enzyme assembly. Genes Dev 13: 2439-2448. doi:10.1101/gad.13.18 .2439

Martin BJE, Chruscicki AT, Howe LJ. 2018. Transcription promotes the interaction of the facilitates chromatin transactions (FACT) complex with nucleosomes in Saccharomyces cerevisiae. Genetics 210: 869-881. doi:10.1534/genetics.118.301349

Mason PB, Struhl K. 2005. Distinction and relationship between elongation rate and processivity of RNA polymerase II in vivo. Mol Cell 17: 831-840. doi:10.1016/j.molcel.2005.02.017

Mayer A, Lidschreiber M, Siebert M, Leike K, Söding J, Cramer P. 2010. Uniform transitions of the general RNA polymerase II transcription complex. Nat Struct Mol Biol 17: 1272-1278. doi:10.1038/nsmb.1903

McCann M, Kellett A, Kavanagh K, Devereux M, Santos AL. 2012. Deciphering the antimicrobial activity of phenanthroline chelators. Curr Med Chem 19: 2703-2714. doi:10.2174/092986712800609733

McCullough L, Connell Z, Petersen C, Formosa T. 2015. The abundant histone chaperones Spt6 and FACT collaborate to assemble, inspect, and maintain chromatin structure in Saccharomyces cerevisiae. Genetics 201: 1031-1045. doi:10.1534/genetics.115.180794

McGinty RK, Tan S. 2016. Recognition of the nucleosome by chromatin factors and enzymes. Curr Opin Struct Biol 37: 54-61. doi:10.1016/j.sbi .2015.11.014

McKnight JN, Boerma JW, Breeden LL, Tsukiyama T. 2015. Global promoter targeting of a conserved lysine deacetylase for transcriptional shutoff during quiescence entry. Mol Cell 59: 732-743. doi:10.1016/j.molcel .2015.07.014

Mews P, Zee BM, Liu S, Donahue G, Garcia BA, Berger SL. 2014. Histone methylation has dynamics distinct from those of histone acetylation in cell cycle reentry from quiescence. Mol Cell Biol 34: 3968-3980. doi:10.1128/MCB.00763-14

Miles S, Li L, Davison J, Breeden LL. 2013. Xbp1 directs global repression of budding yeast transcription during the transition to quiescence and is important for the longevity and reversibility of the quiescent state. PLoS Genet 9: e1003854. doi:10.1371/journal.pgen.1003854

Miller C, Schwalb B, Maier K, Schulz D, Dümcke S, Zacher B, Mayer A, Sydow J, Marcinowski L, Dölken L, et al. 2011. Dynamic transcriptome analysis measures rates of mRNA synthesis and decay in yeast. Mol Syst Biol 7: 458. doi: $10.1038 / \mathrm{msb} .2010 .112$

Musso M, Bianchi-Scarrà G, Van Dyke MW. 2000. The yeast CDP1 gene encodes a triple-helical DNA-binding protein. Nucleic Acids Res 28: 40904096. doi:10.1093/nar/28.21.4090

Myers SA, Wright J, Peckner R, Kalish BT, Zhang F, Carr SA. 2018. Discovery of proteins associated with a predefined genomic locus via dCas9-APEXmediated proximity labeling. Nat Methods 15: 437-439. doi:10.1038/ s41592-018-0007-1

Namdar M, Kearsey SE. 2006. Analysis of Mcm2-7 chromatin binding during anaphase and in the transition to quiescence in fission yeast. Exp Cell Res 312: 3360-3369. doi:10.1016/j.yexcr.2006.07.001

Nemergut ME, Mizzen CA, Stukenberg T, Allis CD, Macara IG. 2001. Chromatin docking and exchange activity enhancement of RCC1 by histones H2A and H2B. Science 292: 1540-1543. doi:10.1126/science 292.5521 .1540

Nourani A, Robert F, Winston F. 2006. Evidence that Spt2/Sin1, an HMGlike factor, plays roles in transcription elongation, chromatin structure, and genome stability in Saccharomyces cerevisiae. Mol Cell Biol 26: 14961509. doi:10.1128/MCB.26.4.1496-1509.2006

O'Duibhir E, Lijnzaad P, Benschop JJ, Lenstra TL, van Leenen D, Groot Koerkamp MJ, Margaritis T, Brok MO, Kemmeren P, Holstege FC. 2014. Cell cycle population effects in perturbation studies. Mol Syst Biol 10: 732 . doi:10.15252/msb.20145172

Panday A, Grove A. 2016. The high mobility group protein HMO1 functions as a linker histone in yeast. Epigen Chrom 9: 13. doi:10.1186/s13072016-0062-8

Pathak R, Singh P, Ananthakrishnan S, Adamczyk S, Schimmel O, Govind CK. 2018. Acetylation-dependent recruitment of the FACT complex and its role in regulating Pol II occupancy genome-wide in Saccharomyces cerevisiae. Genetics 209: 743-756. doi:10.1534/genetics .118 .300943

Porrua O, Libri D. 2015. Transcription termination and the control of the transcriptome: why, where and how to stop. Nat Rev Mol Cell Biol 16: 190-202. doi:10.1038/nrm3943

Prasad R, D'Arcy S, Hada A, Luger K, Bartholomew B. 2016. Coordinated action of Nap1 and RSC in disassembly of tandem nucleosomes. Mol Cell Biol 36: 2262-2271. doi:10.1128/MCB.00195-16

Prasad R, Xu C, Ng DTW. 2018. Hsp40/70/110 chaperones adapt nuclear protein quality control to serve cytosolic clients. J Cell Biol 217: 20192032. doi:10.1083/jcb.201706091

Pufall MA, Kaplan CD. 2013. Mechanisms of eukaryotic transcription. Genome Biol 14: 311. doi:10.1186/gb-2013-14-9-311

Qiu C, Kaplan CD. 2019. Functional assays for transcription mechanisms in high-throughput. Methods 159-160: 115-123. doi:10.1016/j.ymeth 2019.02.017

Radonjic M, Andrau JC, Lijnzaad P, Kemmeren P, Kockelkorn TT, van Leenen D, van Berkum NL, Holstege FC. 2005. Genome-wide analyses reveal RNA polymerase II located upstream of genes poised for rapid response upon S. cerevisiae stationary phase exit. Mol Cell 18: 171-183. doi:10.1016/j.molcel.2005.03.010

Roy KR, Smith JD, Vonesch SC, Lin G, Tu CS, Lederer AR, Chu A, Suresh S, Nguyen M, Horecka J, et al. 2018. Multiplexed precision genome editing with trackable genomic barcodes in yeast. Nat Biotechnol 36: 512-520. doi:10.1038/nbt.4137

Ruone S, Rhoades AR, Formosa T. 2003. Multiple Nhp6 molecules are required to recruit Spt16-Pob3 to form yFACT complexes and to reorganize nucleosomes. J Biol Chem 278: 45288-45295. doi:10.1074/jbc .M307291200

Rutledge MT, Russo M, Belton J-M, Dekker J, Broach JR. 2015. The yeast genome undergoes significant topological reorganization in quiescence. Nucleic Acids Res 43: 8299-8313. doi:10.1093/nar/gkv723

Sagot I, Laporte D. 2019a. The cell biology of quiescent yeast: a diversity of individual scenarios. J Cell Sci 132: jcs213025. doi:10.1242/jcs.213025

Sagot I, Laporte D. 2019b. Quiescence, an individual journey. Curr Genet 65: 695-699. doi:10.1007/s00294-018-00928-w

Sainsbury S, Bernecky C, Cramer P. 2015. Structural basis of transcription initiation by RNA polymerase II. Nat Rev Mol Cell Biol 16: 129-143. doi:10.1038/nrm3952

Schmidtmann E, Anton T, Rombaut P, Herzog F, Leonhardt H. 2016. Determination of local chromatin composition by CasID. Nucleus 7: 476-484. doi:10.1080/19491034.2016.1239000 
Scrutton MC, Wu CW, Goldthwait DA. 1971. The presence and possible role of zinc in RNA polymerase obtained from Escherichia coli. Proc Natl Acad Sci 68: 2497-2501. doi:10.1073/pnas.68.10.2497

Shaw RJ, Reines D. 2000. Saccharomyces cerevisiae transcription elongation mutants are defective in PUR5 induction in response to nucleotide depletion. Mol Cell Biol 20: 7427-7437. doi:10.1128/MCB.20.20.74277437.2000

Shaw RJ, Wilson JL, Smith KT, Reines D. 2001. Regulation of an IMP dehydrogenase gene and its overexpression in drug-sensitive transcription elongation mutants of yeast. J Biol Chem 276: 32905-32916. doi:10 $.1074 /$ jbc.M105075200

Siddiqui K, On KF, Diffley JFX. 2013. Regulating DNA replication in eukarya. Cold Spring Harb Perspect Biol 5: a012930-a012930. doi:10.1101/cshper spect.a012930

Struhl K, Segal E. 2013. Determinants of nucleosome positioning. Nat Struct Mol Biol 20: 267-273. doi:10.1038/nsmb.2506

Sun W, Hola M, Pedley K, Tada S, Blow JJ, Todorov IT, Kearsey SE, Brooks RF. 2000. The replication capacity of intact mammalian nuclei in Xenopus egg extracts declines with quiescence, but the residual DNA synthesis is independent of Xenopus MCM proteins. J Cell Sci 113(Pt 4): 683-695.

Sun M, Schwalb B, Pirkl N, Maier Kerstin C, Schenk A, Failmezger H, Tresch A, Cramer P. 2013. Global analysis of eukaryotic mRNA degradation reveals Xrn1-dependent buffering of transcript levels. Mol Cell 52: 52-62. doi:10.1016/j.molcel.2013.09.010

Swygert SG, Tsukiyama T. 2019. Unraveling quiescence-specific repressive chromatin domains. Curr Genet 65: 1145-1151. doi:10.1007/s00294019-00985-9

Swygert SG, Kim S, Wu X, Fu T, Hsieh T-H, Rando OJ, Eisenman RN, Shendure J, McKnight JN, Tsukiyama T. 2019. Condensin-dependent chromatin compaction represses transcription globally during quiescence. Mol Cell 73: 533-546.e4. doi:10.1016/j.molcel.2018.11.020

Tong AH, Boone C. 2006. Synthetic genetic array analysis in Saccharomyces cerevisiae. Methods Mol Biol 313: 171-192. doi:10.1385/1-59259-958-3 $: 171$

Van Oss SB, Cucinotta CE, Arndt KM. 2017. Emerging insights into the roles of the Paf1 complex in gene regulation. Trends Biochem Sci 42: 788-798. doi:10.1016/j.tibs.2017.08.003

van Welsem T, Korthout T, Ekkebus R, Morais D, Molenaar TM, van Harten K, Poramba-Liyanage DW, Sun SM, Lenstra TL, Srivas R, et al. 2018. Dot1 promotes $\mathrm{H} 2 \mathrm{~B}$ ubiquitination by a methyltransferase-independent mechanism. Nucleic Acids Res 46: 11251-11261. doi:10.1093/nar/ gky801

Vasseur P, Tonazzini S, Ziane R, Camasses A, Rando OJ, Radman-Livaja M. 2016. Dynamics of nucleosome positioning maturation following genomic replication. Cell Rep 16: 2651-2665. doi:10.1016/j.celrep.2016.07 .083

Venters B, Pugh BF. 2009. How eukaryotic genes are transcribed. Crit Rev Biochem Mol Biol 44: 117-141. doi:10.1080/10409230902858785

Verma R, Aravind L, Oania R, McDonald WH, Yates JR 3rd, Koonin EV, Deshaies RJ. 2002. Role of Rpn11 metalloprotease in deubiquitination and degradation by the $26 S$ proteasome. Science 298: 611-615. doi:10 $.1126 /$ science. 1075898

Verzijlbergen KF, van Welsem T, Sie D, Lenstra TL, Turner DJ, Holstege FCP, Kerkhoven RM, van Leeuwen F. 2011. A barcode screen for epigenetic regulators reveals a role for the NuB4/HAT-B histone acetyltransferase complex in histone turnover. PLoS Genet 7: e1002284. doi:10.1371/jour nal.pgen.1002284
Vinayachandran V, Reja R, Rossi MJ, Park B, Rieber L, Mittal C, Mahony S, Pugh BF. 2018. Widespread and precise reprogramming of yeast protein-genome interactions in response to heat shock. Genome Res 28: 357-366. doi:10.1101/gr.226761.117

Vlaming H, Molenaar TM, van Welsem T, Poramba-Liyanage DW, Smith DE, Velds A, Hoekman L, Korthout T, Hendriks S, Altelaar AM, et al. 2016. Direct screening for chromatin status on DNA barcodes in yeast delineates the regulome of H3K79 methylation by Dot1. eLife 5: e18919. doi:10.7554/eLife.18919

Vlaming H, McLean C, Korthout T, Alemdehy MF, Hendriks S, Lancini C, Palit S, Klarenbeek S, Kwesi-Maliepaard EM, Molenaar TM, et al. 2019. Conserved crosstalk between histone deacetylation and H3K79 methylation generates DOT1L-dose dependency in HDAC1-deficient thymic lymphoma. EMBO J 38: e101564. doi:10.15252/embj.2019101564

Vos SM, Farnung L, Boehning M, Wigge C, Linden A, Urlaub H, Cramer P. 2018. Structure of activated transcription complex Pol II-DSIF-PAFSPT6. Nature 560: 607-612. doi:10.1038/s41586-018-0440-4

Wada T, Becskei A. 2017. Impact of methods on the measurement of mRNA turnover. Int J Mol Sci 18: 2723. doi:10.3390/ijms18122723

Weiner A, Chen HV, Liu CL, Rahat A, Klien A, Soares L, Gudipati M, Pfeffner J, Regev A, Buratowski S, et al. 2012. Systematic dissection of roles for chromatin regulators in a yeast stress response. PLoS Biol 10: e1001369. doi:10.1371/journal.pbio.1001369

Wierer M, Mann M. 2016. Proteomics to study DNA-bound and chromatinassociated gene regulatory complexes. Hum Mol Genet 25: R106-R114. doi:10.1093/hmg/ddw208

Wu F, Liu Y, Zhu Z, Huang H, Ding B, Wu J, Shi Y. 2011. The 1.9 Å crystal structure of Prp20p from Saccharomyces cerevisiae and its binding properties to Gsp1p and histones. J Struct Biol 174: 213-222. doi:10.1016/j.jsb .2010 .11 .016

Xie Y, Zheng M, Chu X, Chen Y, Xu H, Wang J, Zhou H, Long J. 2018. Paf1 and Ctr9 subcomplex formation is essential for Paf1 complex assembly and functional regulation. Nat Commun 9: 3795. doi:10.1038/s41467018-06237-7

Xu Y, Bernecky C, Lee CT, Maier KC, Schwalb B, Tegunov D, Plitzko JM, Urlaub H, Cramer P. 2017. Architecture of the RNA polymerase IIPaf1C-TFIIS transcription elongation complex. Nat Commun 8: 15741. doi: $10.1038 /$ ncomms 15741

Yan Z, Costanzo M, Heisler LE, Paw J, Kaper F, Andrews BJ, Boone C, Giaever G, Nislow C. 2008. Yeast barcoders: a chemogenomic application of a universal donor-strain collection carrying bar-code identifiers. Nat Methods 5: 719-725. doi:10.1038/nmeth.1231

Young CP, Hillyer C, Hokamp K, Fitzpatrick DJ, Konstantinov NK, Welty JS Ness SA, Werner-Washburne M, Fleming AB, Osley MA. 2017. Distinct histone methylation and transcription profiles are established during the development of cellular quiescence in yeast. BMC Genomics 18: 107. doi:10.1186/s12864-017-3509-9

Zaidi H, Hoffman EA, Shetty SJ, Bekiranov S, Auble DT. 2017. Second-generation method for analysis of chromatin binding with formaldehyde-cross-linking kinetics. J Biol Chem 292: 19338-19355. doi:10 $.1074 /$ jbc.M117.796441

Zhou H, Liu Q, Shi T, Yu Y, Lu H. 2015. Genome-wide screen of fission yeast mutants for sensitivity to 6-azauracil, an inhibitor of transcriptional elongation. Yeast 32: 643-655. doi:10.1002/yea.3085

Received August 30, 2019; accepted in revised form March 11, 2020.

\section{Genome Research}

www.genome.org 


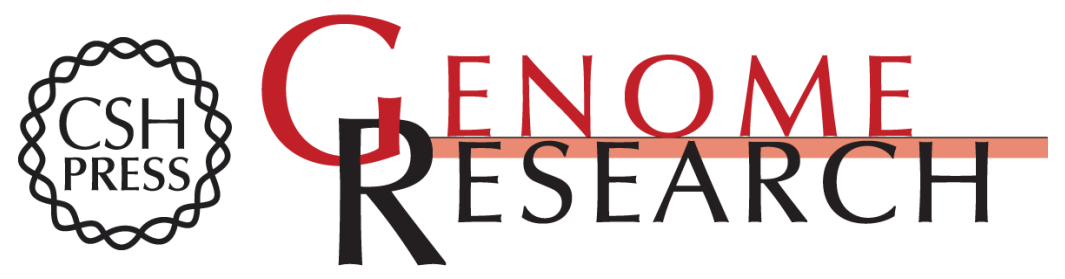

\section{Inhibition of transcription leads to rewiring of locus-specific chromatin proteomes}

Deepani W. Poramba-Liyanage, Tessy Korthout, Christine E. Cucinotta, et al.

Genome Res. 2020 30: 635-646 originally published online March 18, 2020

Access the most recent version at doi:10.1101/gr.256255.119

\section{Supplemental} Material

References

Open Access

Creative Commons License

http://genome.cshlp.org/content/suppl/2020/04/08/gr.256255.119.DC1

This article cites 111 articles, 39 of which can be accessed free at: http://genome.cshlp.org/content/30/4/635.full.html\#ref-list-1

Freely available online through the Genome Research Open Access option.

This article, published in Genome Research, is available under a Creative Commons License (Attribution 4.0 International), as described at http://creativecommons.org/licenses/by/4.0/.

Email Alerting Receive free email alerts when new articles cite this article - sign up in the box at the Service top right corner of the article or click here.

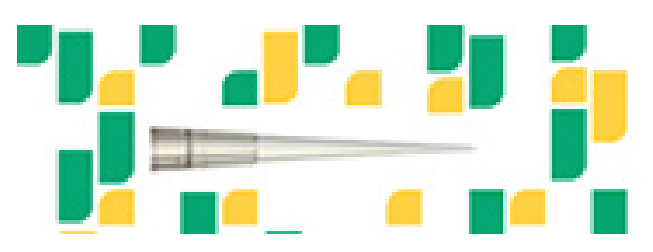

Focused on your science.

\section{गכז}

SCIENTIFIC

To subscribe to Genome Research go to: https://genome.cshlp.org/subscriptions 